21

\title{
Влияние дефектов на поглощение терагерцового излучения в монокристалле $\mathrm{CdSiP}_{2}$
}

\author{
(C) В.С. Ноздрин ${ }^{1}$, С.В. Чучупал ${ }^{1,2}$, Г.А. Командин ${ }^{1}$, В.Н. Курлов ${ }^{3}$, О.Е. Породинков ${ }^{1}$, И.Е. Спектор ${ }^{1}$, \\ Г.M. Катыба ${ }^{1,3,4}$, P.G. Schunemann ${ }^{5}$, K.T. Zawilski ${ }^{5}$ \\ ${ }^{1}$ Институт общей фризики им. А.М. Прохорова РАН, \\ 119991 Москва, Россия \\ ${ }^{2}$ Московский государственный университет им. М.В. Ломоносова, физический факультет, \\ 119991 Москва, Россия \\ ${ }^{3}$ Институт фризики твердого тела РАН, \\ 142432 Черноголовка, Московская обл., Россия \\ ${ }^{4}$ Московский государственный технический университет им. Н.Э. Баумана, \\ 105005 Москва, Россия \\ ${ }^{5}$ BAESystems, Inc., \\ MER15-1813, P.O. Box 868, Nashua, NH03061-0868, USA \\ e-mail: no315@yandex.ru
}

Поступила в редакцию 20.12.2019 г.

В окончательной редакции 22.01.2020 г.

Принята к публикации 28.02.2020 г.

Выполненные в интервале температур от 80 до $300 \mathrm{~K}$ методами терагерцовой (ТГц) импульсной и инфракрасной фурье-спектроскопии измерения спектров пропускания и отражения монокристалла $\mathrm{CdSiP}_{2}$ выявили существенное влияние постростовых дефектов на поглощение в ТГц области частот. Обнаружено, что это поглощение слабо зависит от температуры в отличие от ранее полученных результатов для другого кристалла семейства халькопирита с существенно меньшей концентрацией дефектов. При охлаждении были минимизированы собственные механизмы поглощения и выделен вклад дефектов в поглощение.

Ключевые слова: генерация терагерцового излучения, нелинейно-оптические кристаллы, несобственное поглощение.

DOI: $10.21883 / \mathrm{OS} .2020 .07 .49573 .27-20$

Монокристаллы халькопиритов обладают высокими нелинейно-оптическими коэффициентами [1-3], что в сочетании с хорошими оптическими, механическими и температурными характеристиками определило их широкое применение в качестве оптических параметрических генераторов в инфракрасном (ИК) диапазоне. Их характеристики и параметры описаны в работах [4-8]. Эти кристаллы имеют также хорошие перспективы для работы в качестве генераторов терагерцового (ТГц) излучения [9]. Ввиду того, что генерируемое излучение распространяется непосредственно в кристалле, эффективность работы таких преобразователей существенным образом зависит от поглощения, определяемого в том числе и наличием дефектов. Изучению их влияния на поглощение ИК излучения этими соединениями посвящен ряд работ [10-13]. В отличие от видимого и ИК диапазонов для ТГц области частот этот вопрос изучен достаточно слабо. Причиной этого является сложная суперпозиция различных механизмов, формирующих поглощение в этом диапазоне. В ТГц области частот поглощение формируется как собственными, так и несобственными механизмами, причем несобственное поглощение определяется преимущественно дефектами кристалла и может быть минимизировано совершенствованием технологии выращивания кристалла. Как было показано ранее [14], постростовая обработка кристаллов, например облучение электронами, позволяет в значительной степени снизить потери в области накачки, не вызывая при этом появления в области генерации ТГц излучения дополнительных полос поглощения. Для исследований используются, как правило, кристаллы высокого качества, подвергнутые постростовой обработке. В то же время роль дефектов в формировании общего уровня потерь излучения в ТГц области отдельно не изучалась.

Ввиду того, что дефекты могут образовываться не только во время роста кристалла, но также и в процессе его работы, мы поставили целью нашего исследования определить вклад дефектов в формирование потерь излучения в ТГц области частот, а также изучить механизмы, формирующие поглощение в ТГц диапазоне, и определить их вклады в суммарные диэлектрические потери.

\section{Эксперимент}

Использованный для наших экспериментов кристалл $\mathrm{CdSiP}_{2}$, относящийся к группе тройных полупроводников со структурой халькопирита (пространственная 
группа $D_{2 d}^{12}=I 42 d$, общая формула $\left.\mathrm{A}^{\mathrm{II}} \mathrm{B}^{\mathrm{IV}} \mathrm{C}_{2}^{\mathrm{V}}\right)$, был выращен методом горизонтальной направленной кристаллизации (HGF - horizontal gradient freeze) $[15,16]$. Исследованный образец представлял собой вырезанную из выращенной були плоскопараллельную пластину размером $8 \times 11 \mathrm{~mm}$ и толщиной $0.589 \mathrm{~mm}$, нормаль которой была ориентирована вдоль оси а, а ось с лежала в плоскости среза. Точность определения толщины образца имеет критическое значение при расчете электродинамических параметров по формулам Френеля для плоскопараллельного слоя. Поэтому она была измерена прецизионным микрометром с высокой точностью.

Измерения спектров пропускания выполнены на субмиллиметровом ЛОВ (лампа обратной волны) спектрометре [17] с использованием генератора излучения, перестраиваемого в диапазоне от 3 до $5 \mathrm{~cm}^{-1}$. Спектры пропускания в диапазоне от 5 до $80 \mathrm{~cm}^{-1}$ получены на лабораторном спектрометре с временным разрешением (TDS) [18] в температурном интервале $77-300 \mathrm{~K}$ с шагом $50 \mathrm{~K}$. Для формирования линейно поляризованного излучения в спектрометрах обоих типов использованы периодические структуры из металлических проволок [19], диаметр которых составляет $10 \mu \mathrm{m}$, а период следования $-25 \mu \mathrm{m}$. Такие поляризаторы обеспечивают степень поляризации не хуже $99.9 \%$, а отсутствие подложки исключает дополнительные интерференцию и потери излучения. Спектры отражения в частотном диапазоне $70-4000 \mathrm{~cm}^{-1}$ измерены на ИК фурье-спектрометре BrukerIFS-113v в температурном интервале 77-300 К. При низкотемпературных измерениях был использован криостат оптического типа [20], с целью снижения потерь излучения он был оборудован тонкими полиэтиленовыми окнами. Их применение стало возможным благодаря использованию системы клапанов, соединяющих откачные оптические камеры спектрометров и объем криостата. В результате разность давлений по обеим сторонам окон оставалась незначительной во время всего экспериментального цикла.

Спектры комплексной диэлектрической проницаемости в ТГц области рассчитаны по экспериментальным спектрам пропускания плоскопараллельного образца с использованием формул Френеля.

Определение параметров ИК активных фононов в монокристалле $\mathrm{CdSiP}_{2}$ выполнено по стандартным для ИК спектроскопии дисперсионным моделям гармонического осциллятора:

$$
\begin{gathered}
\mathcal{\varepsilon}^{\prime}(v)=\varepsilon_{\infty}+\sum_{j} \frac{\Delta \varepsilon_{j} v_{j}^{2}\left(v_{j}^{2}-v^{2}\right)}{\left(v_{j}^{2}-v^{2}\right)^{2}+v^{2} \gamma_{j}^{2}}, \\
\varepsilon^{\prime \prime}(v)=\sum_{j} \frac{\Delta \varepsilon_{j} v_{j}^{2} v \gamma_{j}}{\left(v_{j}^{2}-v^{2}\right)^{2}+v^{2} \gamma_{j}^{2}} .
\end{gathered}
$$

Подбором трех подгоночных параметров: диэлектрического вклада $\Delta \varepsilon_{j}$, частоты $v_{j}$ и затухания $\gamma_{j}$ рассчиты- вались модельные спектры отражения

$$
R(v)=\frac{(n(v)-1)^{2}+k^{2}(v)}{(n(v)+1)^{2}+k^{2}(v)},
$$

где $\varepsilon^{\prime}(v)=n^{2}(v)+k^{2}(v), \varepsilon^{\prime \prime}(v)=2 n(v) k(v)$.

\section{Обсуждение}

Спектры пропускания, полученные методами субмиллиметровой ЛОВ-спектроскопии и импульсной широкополосной ТГц спектроскопии, показаны точками на рис. 1. Использование длинноволновой когерентной ЛОВ-спектроскопии для измерения спектров в области $3 \mathrm{~cm}^{-1}$ существенно увеличило достоверность данных на низкочастотном краю диапазона, что позволило количественно проанализировать характер дисперсии. Данные ТГц спектроскопии в силу ряда ограничений [21] не позволяют сделать однозначные выводы о низкочастотных процессах поглощения. Мы выполнили дополнительные измерения с использованием ЛОВ-спектрометра в области $10 \mathrm{~cm}^{-1}$. Полученные в этой области данные показали хорошее взаимное соответствие результатов измерений спектров пропускания этих методик. Основываясь на полном наборе полученных экспериментальных данных, можно утверждать, что основное поглощение в ТГц диапазоне формируется в области $30-40 \mathrm{~cm}^{-1}$ и имеет размытый по частоте характер. Второй важный факт заключается в слабой температурной зависимости этого поглощения. Наиболее выражены изменения на высокочастотном краю диапазона, т.е. вблизи края фононного поглощения. Следует отметить, что данные, полученные нами ранее [22] на другом кристалле, сходны в области фононных резонансов и существенно различаются в ТГц области. Именно это различие и потребовало дополнительного исследования свойств монокристалла.

Для выяснения природы этих изменений и их моделирования мы измерили спектры отражения в области фононных резонансов. Как видно из рис. 2, при понижении температуры пики отражения становятся выше и смещаются в сторону более высоких частот. Это стандартное поведение параметров модельного осциллятора, при котором уменьшается константа затухания и увеличивается его частота. Следует отметить, что определение параметров моды $E(1)$, отмеченной стрелкой на pис. 2, возможно только по спектрам пропускания в ТГц области, поскольку ее вклад $(\Delta \varepsilon \sim 0.014)$ слишком мал. Величина шумов в спектре отражения сопоставима с дисперсионной зависимостью коэффициента отражения, определяемого этой модой, что видно из сравнения модельных спектров с экспериментом. Снижение константы затухания приводит к сужению контура резонанса, как показано на рис. 3 , представляющем расчётные спектры мнимой части диэлектрической проницаемости. Одновременно с сужением контура снижается фононный вклад в потери для ТГц диапазона. 

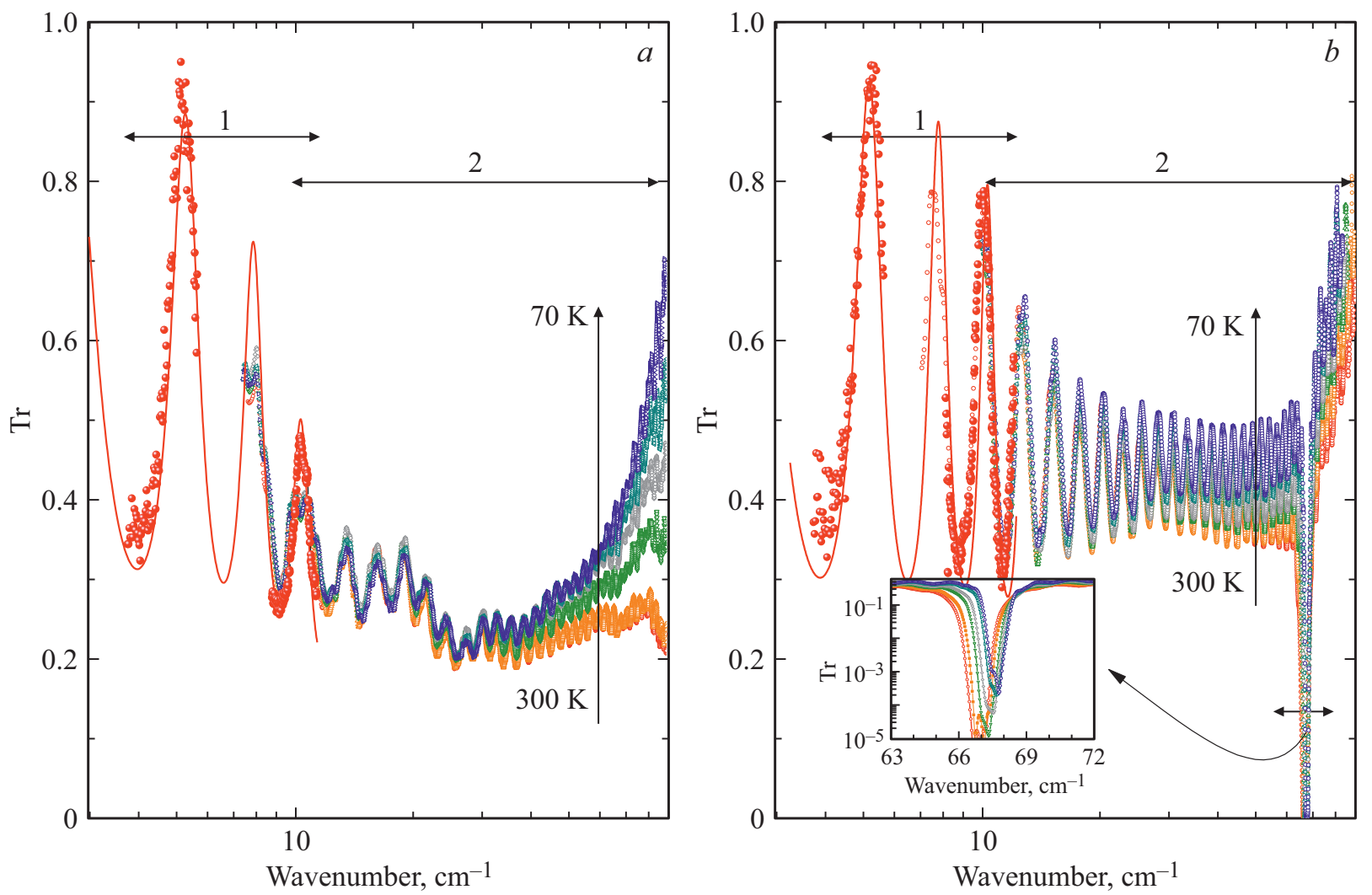

Рис. 1. Эволюция экспериментальных спектров пропускания в ТГц диапазоне при охлаждении от 300 до $70 \mathrm{~K}$. Рисунок $a$ соответствует ориентации поляризации падающего излучения $\mathbf{E} \| \mathbf{c}, b-\mathbf{E} \perp \mathbf{c}$. Крупные точки - данные ЛОВ-спектроскопии, мелкие точки - эксперимент на TDS. Сплошная линия - моделирование эксперимента ЛОВ-спектроскопии по формулам пропускания плоскопараллельного слоя.

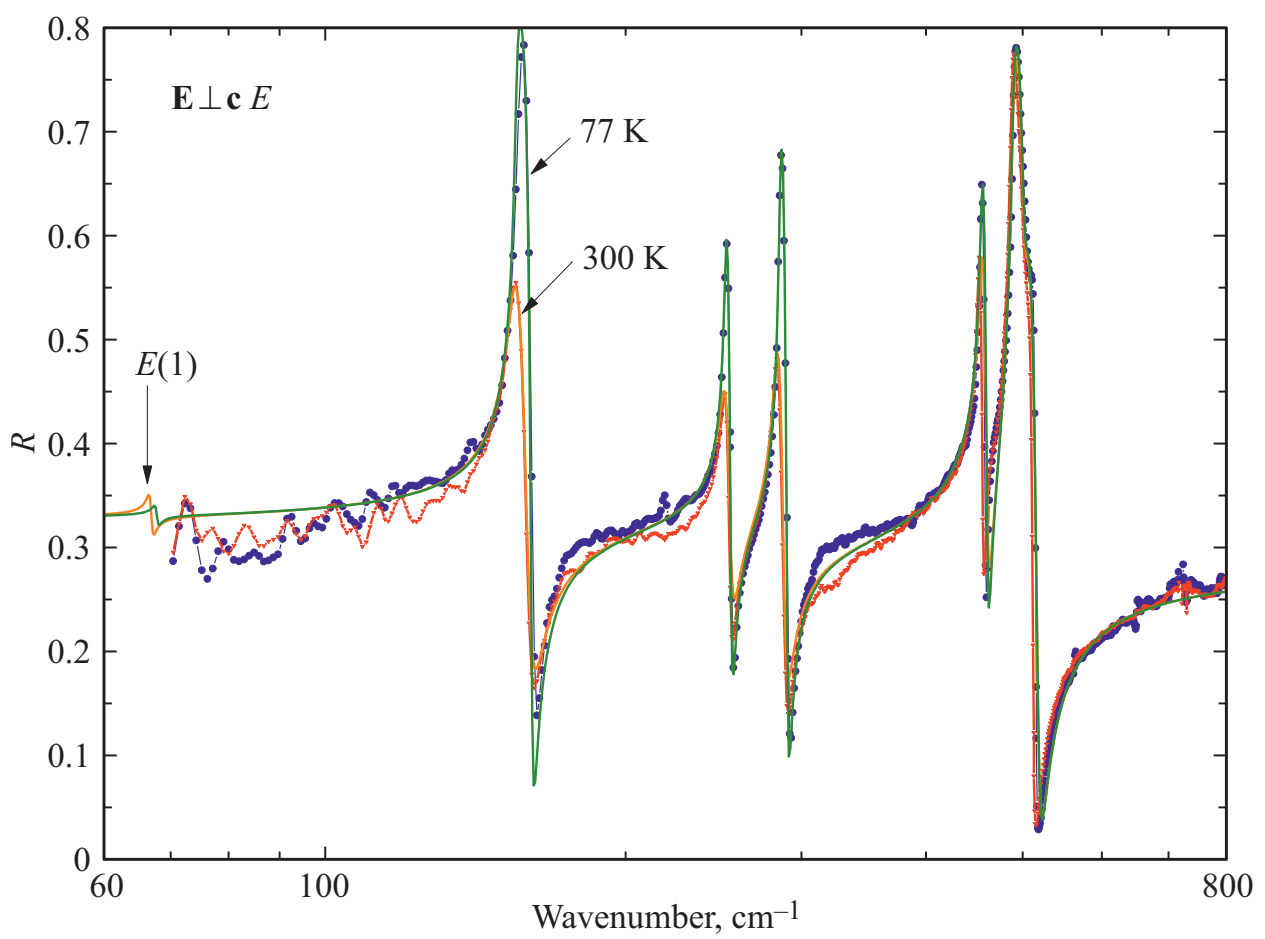

Рис. 2. Спектры отражения монокристалла $\mathrm{CdSiP}_{2}$ при температурах 77 и $300 \mathrm{~K}$ для ориентации поля $\mathbf{E} \perp \mathbf{c}($ моды симметрии $E)$. 


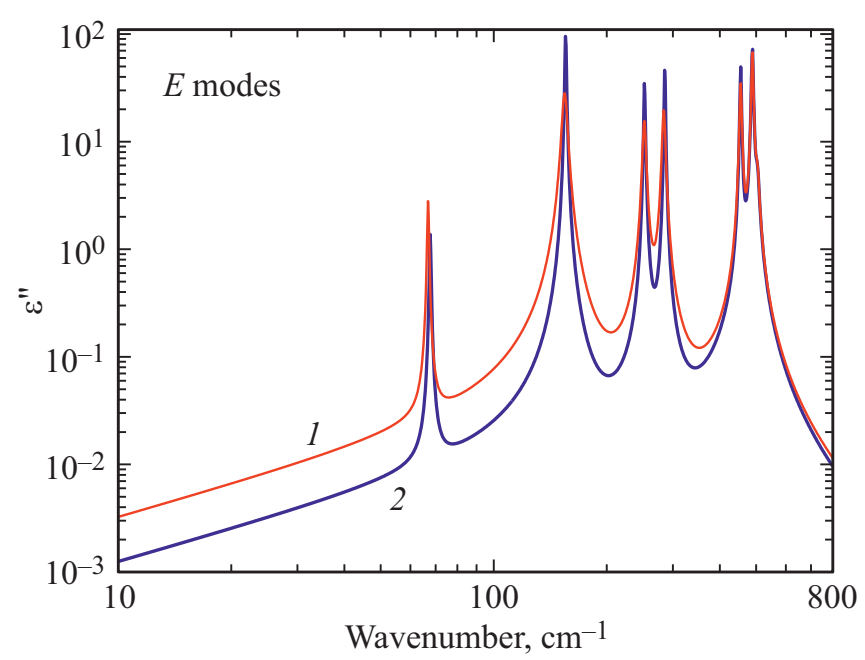

Pис. 3. Спектры мнимой части диэлектрической проницаемости монокристалла $\mathrm{CdSiP}_{2}$ при температурах 300 (линия 1) и $77 \mathrm{~K}$ (линия 2) для ориентации поля $\mathbf{E} \perp \mathbf{c}$ (моды симметрии $E)$.

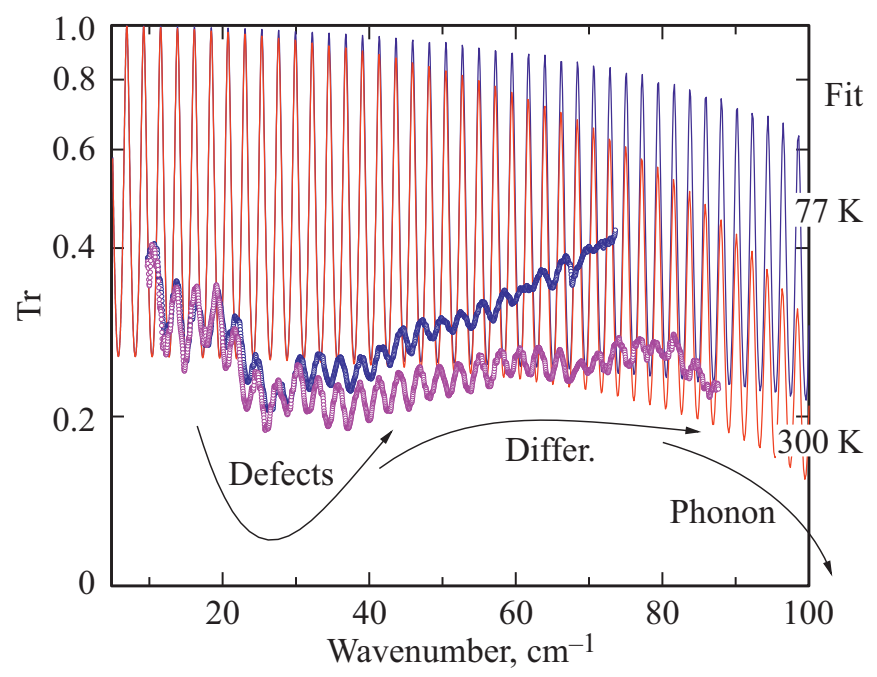

Рис. 4. Расчётные и экспериментальные спектры пропускания монокристалла $\mathrm{CdSiP}_{2}$ в ТГц диапазоне. Стрелками указаны области поглощения на дефектах (Defects), разностных многофононных процессах (Differ.), контуры фононного резонанса (Phonon).

Используя параметры, полученные при моделировании спектров отражения, были рассчитаны спектры пропускания кристалла $\mathrm{CdSiP}_{2}$ с учётом только фононного поглощения (сплошные линии на рис. 4). Расчеты выполнены с учетом многолучевой интерференции для плоскопараллельного образца толщиной $0.589 \mathrm{~mm}$, совпадающей с толщиной измеренного монокристалла. Из рис. 4 видно, что ниже $30 \mathrm{~cm}^{-1}$ пропускание практически не зависит от температуры, а на более высоких частотах при охлаждении наблюдается рост пропускания, обусловленный снижением поглощения на разностных многофононных процессах [23-25]. Фононный вклад отчет- ливо проявляется на высокочастотном краю диапазона в виде спада интерференционной картины.

На поглощение в низкочастотной области могут оказывать влияние несколько факторов. Поскольку $\mathrm{CdSiP}_{2}$ является широкозонным полупроводником, было бы обоснованным предположить наличие проводимости за счет термоактивированных носителей. Однако реализация такого механизма проводимости должна привести к дисперсии друдевского типа, что выразилось бы в снижении коэффициента пропускания на низких частотах при высоких температурах. Но проведенные измерения спектров пропускания с использованием ЛОВ выявили увеличение коэффициента пропускания при понижении частоты, что исключает заметный вклад проводимости в потери. Еще одним фактором могло бы быть несовпадение кристаллографических и главных оптических осей кристалла с направлением поляризации волны ТГц излучения. В этом случае следовало ожидать появление биений на фоне интерференционной картины, зависящей от угла между оптической осью кристалла и направлением поляризации излучения [20]. Однако по данным рентгеноструктурного анализа разориентация оси а кристалла с нормалью к плоскости образца не превышает $3^{\circ}$, и, следовательно, связанные с этим эффекты должны быть меньше чувствительности ТГц метода. Дополнительно проведенные измерения угловой зависимости спектров пропускания от поворота плоскости поляризации с использованием метода, описанного в [20], подтвердили это предположение.

Таким образом, можно утверждать, что уменьшение добротности интерференции и полоса поглощения в области $30 \mathrm{~cm}^{-1}$ в основном связаны с наличием дефектов в данном кристалле.

Вклад поглощения на дефектах в общие потери излучения можно оценить из расчетных спектров $\varepsilon^{\prime \prime}(v)$, приведенных на рис. 5, a. ТГц область для наглядности указана над осью частоты. Суммарный вклад от всех процессов для различных температур показан на этом рисунке линиями 1 и 2. Видно, что при охлаждении образца заметно снижаются потери в высокочастотной области спектра преимущественно за счет сужения контура фононного резонанса и уменьшения поглощения на разностных многофононных переходах. Это и является причиной общего снижения суммарных потерь при охлаждении. Форма контура остается при этом неизменной на низкочастотном краю.

Данные, полученные в работе [22], показывают, что при комнатной температуре потери за счет разностных процессов превышают фононные почти на два порядка, а в ТГц диапазоне величина $\varepsilon^{\prime \prime}$ составляет $10^{-2}$ (зависимости 6 и 7 на рис. 5, a). В тоже время фононный вклад в потери в ТГц области, определенный в данной работе, сопоставим с результатами, полученными в работе [22]. На рис. 5, $b$ приведено сравнение спектров коэффициента поглощения монокристалла $\mathrm{CdSiP}_{2}$, рассчитанных из полученных экспериментальных данных для образцов с большой концентрацией дефектов и с их минимальным 

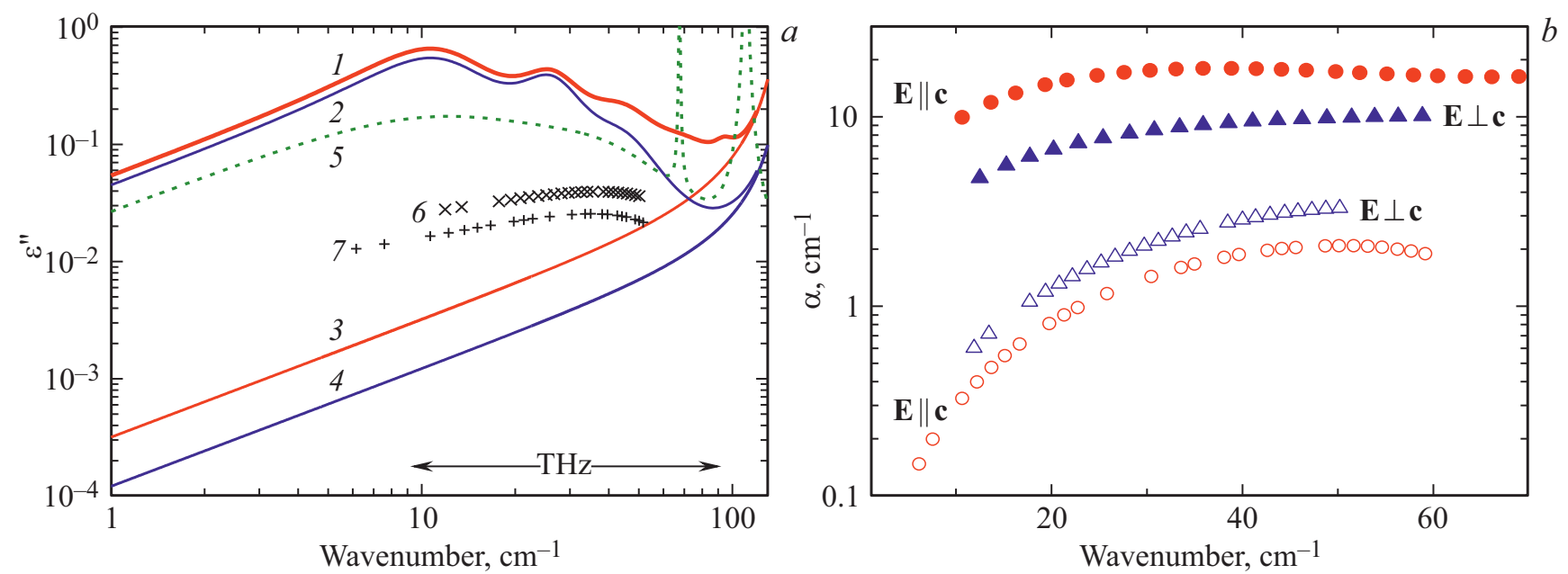

Рис. 5. (a) Спектры мнимой части диэлектрической проницаемости для поляризации $\mathbf{E} \| \mathbf{c}$ (моды симметрии $B_{2}$ ), рассчитанные с учетом всех вкладов в потери для комнатной температуры (1) и для $77 \mathrm{~K}(2)$. Фононный вклад в потери при комнатной температуре $(3)$ и при $77 \mathrm{~K}(4)$. Расчетный спектр $\varepsilon^{\prime \prime}(v)$ для температуры $100 \mathrm{~K}(5)$ в поляризации $\mathbf{E} \perp \mathbf{c}-$ моды симметрии $E$. Для сравнения приведены данные для кристалла с минимальным числом дефектов при $\mathbf{E} \perp \mathbf{c}(6)$ и $\mathbf{E} \| \mathbf{c}(7)$. (b) Частотные зависимости поглощения $\alpha(v)$ в кристалле $\mathrm{CdSiP}_{2}$. Закрашенные символы - кристалл с дефектами, пустые - кристалл с минимальным числом дефектов.

количеством. Видно, что наличие большого числа дефектов на порядок увеличивает поглощение в кристалле, а также уменьшает его дисперсию по сравнению с поглощением в кристалле с минимальным числом дефектов.

Отметим, что роль дефектов в росте потерь максимально проявляется на низкочастотном участке ТГц диапазона и составляет около двух порядков. На высокочастотном краю диапазона заметное снижение потерь может быть получено при понижении температуры кристалла преимущественно за счет уменьшения собственных потерь. При этом в области $50-100 \mathrm{~cm}^{-1}$ потери в охлажденном кристалле сопоставимы по порядку величины с фононным вкладом при комнатной температуре. Вклад в поглощение в этом диапазоне будет давать мода симметрии $E$ с собственной частотой $\sim 67 \mathrm{~cm}^{-1}$, что снижает привлекательность монокристаллов $\mathrm{CdSiP}_{2}$ для использования в качестве источника ТГц излучения.

\section{Заключение}

В результате проведенных исследований установлено, что наличие сравнительно высокой концентрации дефектов дает дополнительный рост поглощения. Суммарные потери от несобственных и многофононных механизмов увеличивают потери не менее чем на три порядка по отношению к фононному вкладу, при этом непосредственно дефекты кристалла дают около двух порядков вклада в $\varepsilon^{\prime \prime}$.

Мы показали, что дефектность кристалла может контролироваться методом ТГц спектроскопии. Это дает возможность не только качественно оценить наличие значительной концентрации дефектов, но также опре- делить его пригодность для использования в качестве активной среды ТГц диапазона.

Также установлено, что охлаждение монокристалла $\mathrm{CdSiP}_{2}$ до 80-100 K минимизирует собственные потери, что приводит к увеличению эффективности его работы как нелинейно-оптического преобразователя излучения. Однако необходимо отметить, что наличие моды симметрии $E$ с частотой около $67 \mathrm{~cm}^{-1}$ сужает частотный диапазон его использования.

\section{Финансирование работы}

Работа выполнена при финансовой поддержке Российского фонда фундаментальных исследований, грант № 18-32-00322.

\section{Конфликт интересов}

Авторы заявляют, что у них нет конфликта интересов.

\section{Список литературы}

[1] Carnio B.N., Schunemann P.G., Zawilski K.T., Elezzabi A.Y.// Opt. Lett. 2017. V. 42. N 19. P. 3920-3923. doi 10.1364/OL.42.003920

[2] Piyathilaka H.P., Sooriyagoda R., Dewasurendra V., Johnson M.B., Zawilski K.T., Schunemann P.G., Bristow A.D. // Opt. Expr. 2019. V. 27. N 12. P. 16958-16965. doi 10.1364/OE.27.016958

[3] Itoh N., Fujinaga T., Nakau T. // Jap. J. Appl. Phys. 1978. V. 17. N 5. P. 951-952. doi 10.1143/JJAP.17.951

[4] Vodopyanov K.L., Ganikhanov F., Maffetone J.P., Zwieback I., Ruderman W. // Opt. Lett. 2000. V. 25. N 11. P. 841-843. doi 10.1364/OL.25.000841 
[5] Lambrecht W.R.L., Jiang X. // Phys. Rev. B. 2004. V. 70. P. 045204. doi 10.1103/PhysRevB.70.045204

[6] Kemlin V., Brand P., Boulanger B., Segonds P., Schunemann P.G., Zawilski K.T., Menaert B., Debray J. // Opt. Lett. 2011. V. 36. N 10. P. 1800-1802. doi 10.1364/OL.36.001800

[7] Kemlin V., Boulanger B., Petrov V., Segonds P., Menaert B., Schunemann P.G., Zawilski K.T. // Opt. Materials Express. 2011. V. 1. N 7. P. 1292-1300. doi 10.1364/OME.1.001292

[8] Marchev G., Tyazhev A., Petrov V., Schunemann P.G., Zawilski K.T., Stoppler G., Eichhorn M. // Lasers, Sources, and Related Photonic Devices. OSA Technical Digest (CD) (Optical Society of America, 2012). Paper AM1A.3. doi 10.1364/ASSP.2012.AM1A.3

[9] Rowley J.D., Bas D.A., Zawilski K.T., Schunemann P.G., Bristow A.D. // J. Opt. Soc. Am. B. 2013. V. 30. N 11. P. 2882-2888. doi 10.1364/JOSAB.30.002882

[10] Zhang G., Tao X., Wang S., Liu G., Shi Q., Jiang M. // J. Crystal Growth. 2011. V. 318. Is. 1. P. 717-720. doi 10.1016/j.jcrysgro.2010.11.038

[11] Scherrer E.M., Kananen B.E., Golden E.M., Hopkins F.K., Zawilski K.T., Schunemann P.G., Halliburton L.E., Giles N.C. // Optical Materials Express. 2017. V. 7. N 3. P. 658-664. doi 10.1364/OME.7.000658

[12] Brudnyi V.N., Budnitskii D.L., Krivov M.A., Masagutova R.V., Prochukhan V.D., Rud Yu.V. // Physica Status Solidi (a). 1978. V. 50. N 2. P. 379-384. doi 10.1002/pssa.2210500202

[13] Giles N.C., Bai L., Chirila M.M., Garces N.Y., Stevens K.T., Schunemann P.G., Setzler S.D., Pollak T.M. // J. Appl. Phys. 2003. V. 93. N 11. P. 8975-8981. doi 10.1063/1.1572195

[14] Chuchupal S.V., Komandin G.A., Zhukova E.S., Porodinkov O.E., Spektor I.E., Gribenyukov A.I. // Phys. Solid State. 2015. V. 57. N 8. P. 1607-1612. doi $10.1134 / \mathrm{S} 1063783415080041$

[15] Schunemann P.G., Zawilski K.T. US Patent No 8379296, 2013.

[16] Zawilski K.T., Schunemann P.G., Pollak T.C., Zelmon D.E., Fernelius N.C., Hopkins F.K. // J. Cryst. Growth. 2010. V. 312. Is. 8. P. 1127-32. doi 10.1016/j.jcrysgro.2009.10.034

[17] Kozlov G., Volkov A. // Topics in Appl. Phys. 1998. V. 74. P. 51-109. doi 10.1007/BFb0103420

[18] Командин Г.А., Гавдуш А.А., Гончаров Ю.Г., Породинков О.Е., Ноздрин В.С., Чучупал С.В., Спектор И.Е. // Опт. и спектр. 2019. Т. 126. № 5. С. 596-603. doi 10.21883/OS.2019.05.47658.7-19; Komandin G.A., Gavdush A.A., Goncharov Yu.G., Porodinkov O.E., Nozdrin V.S., Chuchupal S.V., Spektor I.E. // Opt. Spectrosc. 2019. V. 126. Is. 5. P. 514-522. doi 10.1134/S0030400X1905014X

[19] Volkov A.A., Gorshunov B.P., Irisov A.A., Kozlov G.V., Lebedev S.P. // Intern. J. Infrared and Millimeter Waves. 1982. V. 3. N 1. P. 19-43. doi 10.1007/BF01007199

[20] Komandin G.A., Anzin V.B., Ulitko V.E., Gavdush A.A., Mukhin A.A., Goncharov Y.G., Porodinkov O.E., Spektor I.E. // Opt. Engin. 2019. V. 59. N 6. P. 061603. doi 10.1117/1.OE.59.6.061603

[21] Guliano B.M., Gavdush A.A., Muller B., Zaitsev K.I., Gassi T., Palumbo M.E., Baratta G.A., Scire S., Komandin G.A., Yurchenko S.O., Casseli P. // Astronomy and Astrophysics. 2019. V. 629. A112. doi 10.1051/0004-6361/201935619

[22] Komandin G.A., Chuchupal S.V., Goncharov Y.G., Porodinkov O.E., Spektor I.E., Zawilski K.T., Schunemann P.G. // Materials Research Express. 2019. V. 6. N 2. P. 026204. doi 10.1088/2053-1591/aaeec6
[23] Stolen R., Dransfeld K. // Phys. Rev. 1965. V. 139. N 4A. P. 1295-1303. doi 10.1103/PhysRev.139.A1295

[24] Sparks M., King D.F., Mills D.L. // Phys. Rev. B. 1982. V. 26. N 12. P. 6987-7003. doi 10.1103/PhysRevB.26.6987

[25] Komandin G.A., Porodinkov O.E., Spektor I.E., Volkov A.A. // Phys. Solid State. 2009. V. 51. N 10. P. 2045-2050. doi $10.1134 / \mathrm{S} 1063783409100096$ 